\title{
openheart Exercise dose and all-cause mortality within extended cardiac rehabilitation: a cohort study
}

\author{
Claire Taylor, ${ }^{1}$ Costas Tsakirides, ${ }^{1}$ James Moxon, ${ }^{2}$ James W Moxon, ${ }^{3}$ \\ Michael Dudfield, ${ }^{4}$ Klaus Witte, ${ }^{5}$ Lee Ingle, ${ }^{6}$ Sean Carroll ${ }^{6}$
}

\begin{abstract}
- Additional material is published online only. To view please visit the journal online (http://dx.doi.org/10.1136/ openhrt-2017-000623)
\end{abstract}

To cite: Taylor C, Tsakirides C, Moxon J, et al. Exercise dose and all-cause mortality within extended cardiac rehabilitation: a cohort study. Open Heart 2017;4:e000623. doi:10.1136/ openhrt-2017-000623

Received 28 February 2017 Revised 28 May 2017

Accepted 20 June 2017

\section{(a) CrossMark}

${ }^{1}$ Carnegie School of Sport,Leeds Beckett University, Leeds, UK ${ }^{2}$ Burton Croft Surgery, Leeds, UK ${ }^{3}$ Retired General Practitioner, Leeds, UK

${ }^{4}$ Leeds Leisure Services,Leeds City Council, Leeds, UK

${ }^{5}$ Division of Cardiovascular and Diabetes Research, University of Leeds, Leeds, UK

${ }^{6}$ Department of Sport, Health and Exercise Science, University of Hull, Hull, UK

Correspondence to Claire Taylor, CarnegieSchool of Sport, Leeds Beckett University, Leeds, LS6 3QS, UK; c.I.taylor@ leedsbeckett.ac.uk

\section{ABSTRACT}

Aims To investigate the relationship between exercise participation, exercise 'dose' expressed as metabolic equivalent (MET) hours (h) per week, and prognosis in individuals attending an extended, community-based exercise rehabilitation programme.

Methods Cohort study of 435 participants undertaking exercise-based cardiac rehabilitation (CR) in Leeds, West Yorkshire, UK between 1994 and 2006, followed up to 1 November 2013. MET intensity of supervised exercise was estimated utilising serial submaximal exercise test results and corresponding exercise prescriptions. Programme participation was routinely monitored. Cox regression analysis including time-varying and propensity score adjustment was applied to identify predictors of long-term, all-cause mortality across exercise dose and programme duration groups.

Results There were 133 events (31\%) during a median follow-up of 14 years (range, 1.2 to 18.9 years). The significant univariate association between exercise dose and all-cause mortality was attenuated following multivariable adjustment for other predictors, including duration in the programme. Longer-term adherence to supervised exercise training (>36 months) was associated with a $33 \%$ lower mortality risk (multivariate-adjusted HR: $0.67 ; 95 \% \mathrm{Cl}: 0.47$ to $0.97 ; p=0.033$ ) compared with all lesser durations of CR (3, 12, 36 months), even after adjustment for baseline fitness, comorbidities and survivor bias.

Conclusion Exercise dose (MET-h per week) appears less important than long-term adherence to supervised exercise for the reduction of long-term mortality risk. Extended, supervised CR programmes within the community may play a key role in promoting long-term exercise maintenance and other secondary prevention therapies for survival benefit.

\section{INTRODUCTION}

Cardiac rehabilitation (CR) has been shown to reduce recurrent cardiovascular disease (CVD) and all-cause mortality in individuals with coronary heart disease (CHD), independently of other risk factors. Two landmark observational studies using large, administrative databases demonstrated a significant association between the cumulative number of CR sessions attended and this mortality

\section{KEY MESSAGES}

What is already known about this subject?

- Existing clinical trial or observational epidemiological evidence has not fully explored the role of exercise 'dose'(frequency, intensity and duration), as a mechanism for reducing mortality risk in patients undertaking structured, supervised exercise-based cardiac rehabilitation (CR).

What does this study add?

- Long-term participation (greater than 36 months) in supervised CR exercise within an extended, community-based maintenance programme is associated with a significant stepwise survival benefit compared to all shorter CR durations, irrespective of fitness level at programme entry.

How might this impact on clinical practice?

- These findings provide support for clinician endorsement of extended, supervised exercise rehabilitation for individuals completing early outpatient CR. Significant survival benefit may be achieved through long-term maintenance programmes within the community.

risk reduction, ${ }^{12}$ and applied multiple analytical techniques to limit confounding. These studies reported 'dose-response' benefits of CR from both dichotomous and continuous measures, however, the strength of this suggested dose-response association has not been replicated across subsequent cohort studies. $^{3-5}$

Stratified analyses of higher versus lower exercise doses in consecutive meta-analyses of CR have shown no significant association with all-cause mortality. ${ }^{67}$ Yet, the composite measure of exercise dose used in these analyses comprised only frequency and duration of CR exercise and did not include an integral component of exercise dose; exercise intensity. Intensity is a reliable predictor of cardiorespiratory fitness gain, ${ }^{8}$ a strong prognostic indicator in patients with CHD. ${ }^{9-11}$ Furthermore, trials included in meta-analyses 
were limited to both short duration exercise interventions (typically 12 to 18 weeks and up to 36 sessions) and survival follow-up (median 12 months). ${ }^{7}$

It is apparent that existing clinical trial or observational epidemiological evidence has not fully explored the role of exercise dose and its integral components (exercise intensity, frequency and duration) as a mechanism for reduced all-cause mortality risk in patients undertaking structured, supervised exercise-based CR. No known UK-based CR study has reported on this relationship. Recent studies emphasise the need to examine this dose-response relationship ${ }^{12}$ and to rectify CR programme deficiencies with alternative secondary prevention models. Accordingly, the aim of this study was to examine exercise dose and prognosis in a representative CHD cohort undertaking long-term, supervised CR within the community.

\section{METHODS}

\section{Study population}

This is a cohort study of participants entering a community-based CR exercise programme, 'Heart Watch' delivered by local council leisure services in Leeds, UK, between 3 January 1994 and 16 October 2006. Participants were enrolled with a clinical diagnosis of CHD, were clinically stable and discharged from hospital for a minimum of 12 weeks. Study ethical approval was obtained from Leeds East Local Research Ethics Committee (study reference: 13YH0099) and permission to access data from medical records without consent granted by the Health Research Authority Confidentiality Advisory Group (study ref: CAG5-03[PR6]2013). The primary outcome measure was all-cause mortality at 14 years. Survival status was determined from clinical databases (Leeds Teaching Hospitals' NHS Trust) using full name, date of birth and postcode to provide data linkage with the Heart Watch registry.

\section{Description of CR programme}

The exercise training component of the Heart Watch CR programme has been fully described elsewhere.$^{10} \mathrm{It}$ is an ongoing maintenance programme centred on supervised exercise training in a community setting up to 5 days per week. Briefly, participants received mixed circuit-based exercise of $24 \mathrm{~min}$ with appropriate warm up and cool down. On the basis of resting heart rate (HR), final HR and rating of perceived exertion (RPE) from baseline and consecutive exercise tests, participants were prescribed individualised target HR exercise training (up to $85 \%$ of age-predicted maximum HR).

\section{Assessment of test variables}

Indices of submaximal cardiorespiratory fitness (sCRF) were obtained from submaximal exercise testing at programme entry and subsequent re-tests (initial re-test at 12 weeks and annually, thereafter). The majority of exercise tests were conducted using a progressive incremental treadmill walking protocol designed for use in patients with lower levels of functional capacity. ${ }^{13}$ Participants exercised up to a pre-specified test termination criteria (in the region of $85 \%$ age-predicted maximal HR or RPE 16 , unless clinically contraindicated. ${ }^{14}$ Directly-determined $\mathrm{VO}_{2}$ and $\mathrm{HR}$ values for corresponding stages of this protocol on the treadmill and cycle ergometer have been shown to be well-correlated $(r=0.94$ and $r=0.89$, respectively), indicating comparability between modalities for the small number of participants tested on a cycle ergometer. ${ }^{13}$ Handrail support during treadmill testing was discouraged. Participants' medication status and test modality were consistent between exercise tests.

CR workload was described in estimated metabolic equivalents (MET; where 1 MET $\sim 3.5 \mathrm{~mL} \mathrm{O}_{2} / \mathrm{kg} / \mathrm{min}$ ). Estimated METs were calculated from the oxygen consumption requirements of the final workload during sequential submaximal exercise tests using established equations, ${ }^{1516}$ which corresponded with prescribed exercise (upper training range $\sim 75 \% \mathrm{HRR}$ ). Mean estimated MET intensity of CR exercise training was multiplied by mean weekly duration (hours) and mean frequency of supervised exercise to produce an estimate of MET hours [h] per week (ie, mean MET intensity $x$ mean session duration $[\mathrm{h}] \mathrm{x}$ mean weekly frequency). As there are no established thresholds for presenting MET-h, median values are presented with participants divided into lower and higher exercise dose groups $(\leq 6.6$ MET-h per week and $>6.6$ MET-h per week, respectively), congruent with previous literature.

The cutpoints used to define programme duration were 3,12 and 36 months, reflecting typically reported CR durations and a longer-term ( $>36$ months) intervention exceeding most existing RCT evidence. ${ }^{7}$ Cutpoints for baseline sCRF categories (low, moderate, higher) were derived from sex-specific distributions of exercise test time congruent with previous literature. ${ }^{11} 17$ Comorbid status was assessed using a modified Charlson comorbidity score. ${ }^{18}$ Baseline test date was used to adjust for temporal trends in CHD management. Adherence to supervised exercise and habitual physical activity were obtained from attendance registers and self-reported by participants during interview at each re-test. Self-reported physical activity and inactivity codes were congruent with those applied in the British Regional Heart Study. ${ }^{19}$ Activity codes were therefore a relative measure of physical activity, not a measure of the total time spent in activity.

\section{Statistical analysis}

Measures of central tendency and dispersion are reported as mean and SD unless specified and discrete data as counts and percentages. The Independent samples t-test or Mann-Whitney $U$ test (continuous data) and Fishers Exact test (categorical data) were used for comparisons between groups, as appropriate.

A propensity score was derived from binary logistic regression analysis, using exercise dose as the outcome to determine the probability that a participant would undergo an above median dose ( $>6.6$ MET-h per week) 
of supervised exercise training. This propensity score was used to adjust for selection bias attributable to non-randomised assignment of exercise dose and baseline variables included are provided in supplementary online material (see online supplementary table 1). Backward stepwise elimination was employed and resultant independent predictors used to calculate propensity score.

A parsimonious Cox proportional-hazards (backward stepwise) model was used to assess the effect of variables on survival. Proportionality of hazards were tested using a $\log (-\log$ (survival) $)$ plot versus $\log$ (time) plot and partial residuals. Multicollinearity was assessed using variance inflation factors. Prognostic factors statistically significant at the 5\% level in univariate analyses were entered into multivariable Cox models. Extended Cox regression models were constructed to account for non-proportionality in conventional models. The time axis was partitioned at the point where a distinct change in the slope of the hazard was observed (at 9years) and hazard ratios were then estimated for each time interval independently. ${ }^{20}$ Multivariate Cox regression models were adjusted for confounders, significant univariate predictors of all-cause death and individual propensity scores. To account for time-dependent 'survivor' bias $^{21}$ programme duration was also analysed as a time-dependent covariate. KaplanMeier analysis (log-rank test) was applied to verify the time-dependent occurrence of death in groups stratified by exercise dose. A mixed model two-way ANOVA was used to assess changes in SCRF indices across groups. For variables of primary interest data were complete and missing data in other variables accounted for $<5 \%$ of the final sample. SPSS 23.0 (IBM SPSS, USA) was used for all analyses.

\section{RESULTS}

\section{Participant characteristics}

A total of 994 participants were enrolled to CR. Those who died within 12 months of their baseline assessment $(n=7)$; without complete follow-up $(n=315)$; or without a valid baseline exercise test $(n=2)$ were excluded from the analysis of cohort characteristics at baseline $(n=670)$. Follow-up was complete to 1 November 2013. Two-hundred and three participants (30\%) in the CR cohort did not return for initial CR re-assessment scheduled at 12 weeks however were similar to completers with respect to survival over 14 years (age-adjusted HR: 1.10; $95 \%$ CI: 0.82 to 1.48 ; log rank $\mathrm{p}=0.520$ for non-completers compared with completers). For the assessment of CR exercise dose, non-completers $(n=203)$ and participants without a valid measure of weekly exercise training frequency $(n=32)$ were excluded, leaving a final study population of $\mathrm{n}=435$. Baseline participant characteristics are displayed in supplementary material online, see online supplementary table S2 and S3.

Participants undertaking a lower volume of supervised exercise underwent a median 4.9 MET-h per week (interquartile range [IQR] 3.3 to 5.6 MET-h) during 30 months of CR (IQR 3 to 85 months). Those undertaking a higher volume underwent a median 8.4 MET-h per week (IQR 7.3 to 10.0 MET-h; $p<0.0005)$ during 42 months of CR (IQR 3 to 100 months; $\mathrm{p}=0.070$ ). The frequency of supervised exercise for lower and higher exercise dose groups was 2 (IQR 1 to 2) sessions per week and 3 (IQR 2 to 3) sessions per week $(\mathrm{p}<0.0005)$, respectively. Those undertaking a lower volume of supervised exercise were older, more likely to be female, less physically active, had lower sCRF levels (estimated METs) and attained a higher \% of age-predicted maximum exercise HR on baseline testing. They were also more likely to be using diuretic therapy and less likely to have a prescription for antiplatelet therapy. Median propensity scores were consistent with differences in baseline characteristics between exercise dose groups: 0.54 (IQR: 0.40 to 0.69 ) and 0.63 ([IQR: 0.55 to 0.73 ]; $\mathrm{p}<0.0005)$, respectively.

Thirty-one per cent of the entire cohort died $(n=133)$ during a median follow-up of 14 years (range, 1.2 to 18.9 years). Significant univariate predictors of all-cause mortality were: age, baseline sCRF, duration in the programme, modified Charlson comorbidity index score, taking specific cardioprotective medication (diuretic, statin, ACE-inhibitor, calcium channel blocker and antiplatelet therapies), diabetes, TC/HDL-c ratio, date of CR programme entry and employment status. Exercise dose (MET-h per week) treated as a continuous variable was inversely associated with all-cause mortality (HR 0.94; $95 \%$ CI 0.89 to $1.00 ; p=0.05)$. Sex, smoking status, previous MI, $\beta$-blocker use, digoxin use, hypertension, BMI, self-reported physical inactivity, family history, indices of multiple social deprivation and test modality were not significant univariate predictors in this cohort.

MET-h of supervised CR exercise was associated with an unadjusted HR of 0.70 (95\% CI, 0.50 to $0.98 ; \mathrm{p}=0.038$ ) and significantly lower all-cause mortality on KaplanMeier analysis (figure 1) (online supplementary figure S1compares CR completers stratified by exercise dose with non-completers). With evidence of late separation of event curves and non-constant risk during follow-up (confirmed in log-minus-log plots), early and late follow-up periods were analysed independently. Ttable 1 displays results from the extended multivariate Cox regression models.

Following multivariable adjustment for confounding variables and propensity score, the strongest prognostic factors for all-cause mortality were: duration in the CR programme, age, baseline sCRF and secondary prevention medication use (ACE inhibitor, calcium channel blocker, statin and diuretic), respectively. The univariate association between exercise dose and mortality was not found to be independent of these factors (propensity score-adjusted HR: 1.14; $95 \%$ CI: 0.74 to 1.76 ).

Multivariable model 1: adjusted for significant univariate predictors (age, sCRF, diuretic, statin, ACE-inhibitor, calcium channel blocker use, modified Charlson comorbidity index, date of first test, duration in CR programme, diabetes and employment status. 


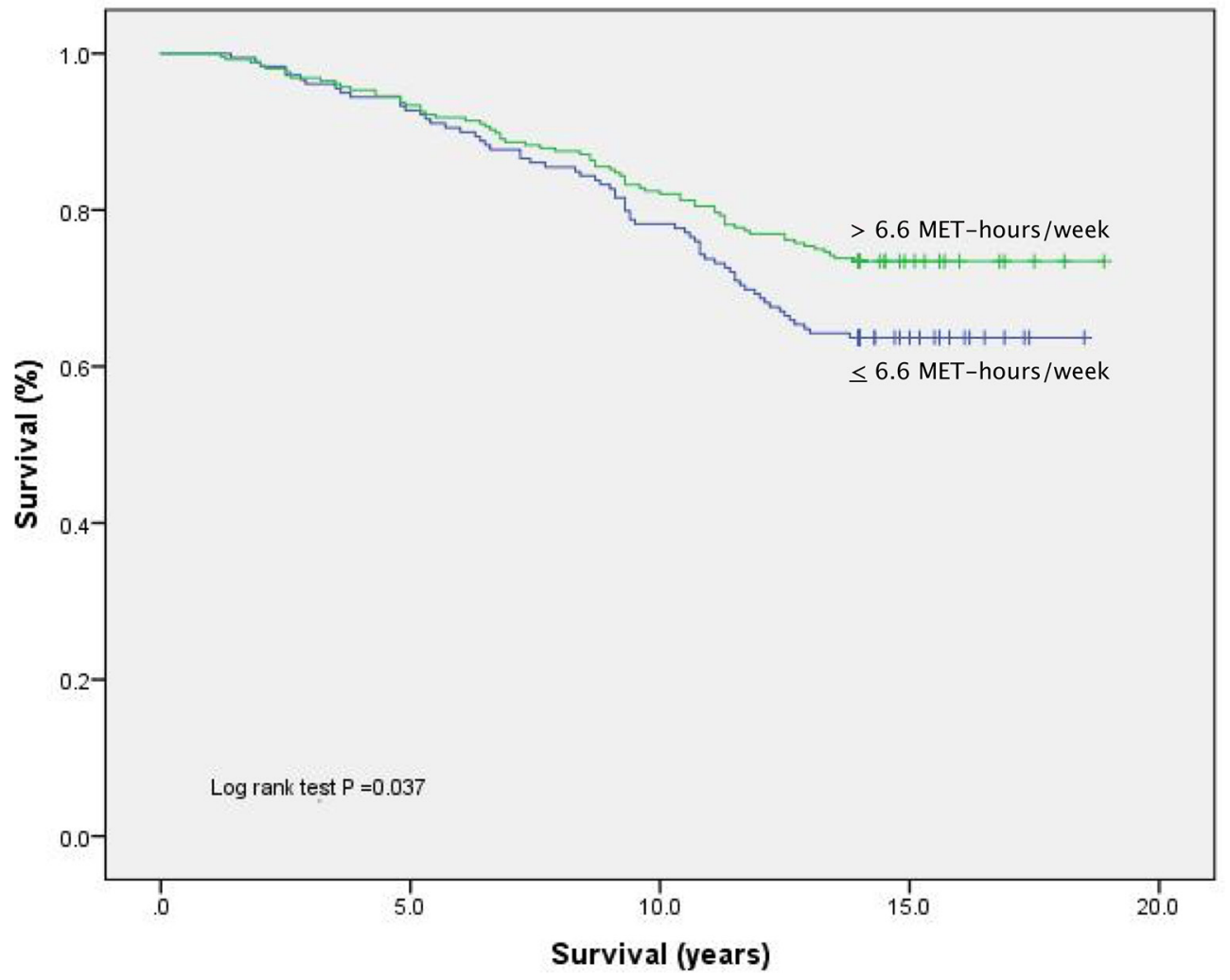

Figure 1 Unadjusted Kaplan-Meier plot comparing CR participants dichotomised on the basis of CR exercise dose undertaken (MET-h per week).

Multivariable model 2: adjusted for propensity score (age, sex, sCRF, ACE-inhibitor, $\beta$-Blocker use and BMI) and other significant univariate predictors: duration in programme, statin, diuretic, calcium channel blocker, antiplatelet therapy use, modified Charlson comorbidity index, date of first test, diabetes, TC/HDL-c ratio and employment status

Undertaking $>6.6$ MET-h supervised exercise training was associated with a lower risk of mortality after $>9$ years follow-up, however risk estimates were attenuated.

There were no statistically significant interactions between exercise dose and changes in indices of sCRF. All participants completing CR improved their exercise test duration $(p<0.0005)$ between first and final re-test: $>6.6$ MET-h per week $(1.5 \pm 2.7 \mathrm{~min})$ and $\leq 6.6 \mathrm{MET}-\mathrm{h}$ per week $(1.3 \pm 2.3 \mathrm{~min})$. Similarly, there were no clinically meaningful differences between groups with respect to change in resting or peak HR.
Given the strong prognostic influence of long-term programme adherence in this cohort, stratified analysis by programme adherence (up to 3, 12 and 36 months and $>36$ months supervised exercise) was also performed. figure 2 illustrates the cumulative event rates for these short-term, extended and long-term CR groups.

Undertaking more than 36 months supervised exercise was associated with a $51 \%$ lower risk of death from all causes over 14 years (multivariate-adjusted HR: 0.49; 95\% CI: 0.34 to $0.72 ; \mathrm{p}<0.0005)$, compared with all other durations of CR: $48 \%$ lower compared with participants adhering up to 36 months (multivariate-adjusted HR: 0.52 ; $95 \%$ CI: 0.31 to $0.87 ; \mathrm{p}=0.013)$; and $60 \%$ lower compared with those adhering up to 12 months (multivariate-adjusted HR: 0.40 ; $95 \%$ CI: 0.23 to $0.69 ; \mathrm{p}=0.001$ ). The risk estimate compared with participants undertaking short-term CR (up to 3 months) and accounting for non-proportional hazards during follow-up was

Table 1 Estimated hazard ratios $(95 \% \mathrm{Cl})$ for all-cause mortality based on supervised exercise dose (MET-h per week) in independent Cox models for two time periods

\begin{tabular}{|c|c|c|c|c|c|c|}
\hline \multirow[t]{2}{*}{ Model } & \multicolumn{3}{|c|}{ Event time $<9$ years } & \multicolumn{3}{|c|}{ Event time $>9$ years } \\
\hline & HR $^{*}$ & $95 \% \mathrm{Cl}$ & $P$ Value & HR & $95 \% \mathrm{Cl}$ & $P$ Value \\
\hline Unadjusted & 0.85 & 0.53 to 1.36 & 0.492 & 0.57 & 0.35 to 0.92 & 0.023 \\
\hline Multivariate Model 1 & 1.67 & 1.00 to 2.88 & 0.049 & 1.02 & 0.60 to 1.73 & 0.939 \\
\hline Multivariate Model 2 (propensity score) & 1.32 & 0.76 to 2.30 & 0.322 & 0.98 & 0.56 to 1.72 & 0.949 \\
\hline
\end{tabular}

${ }^{*} \mathrm{HR}$ for exercise volume $>6.6 \mathrm{MET}-\mathrm{h}$ per week ( $\leq 6.6$. MET-h per week is reference category) Time partitioning at 9 years corresponds to a distinct change in the slope of the hazard. 


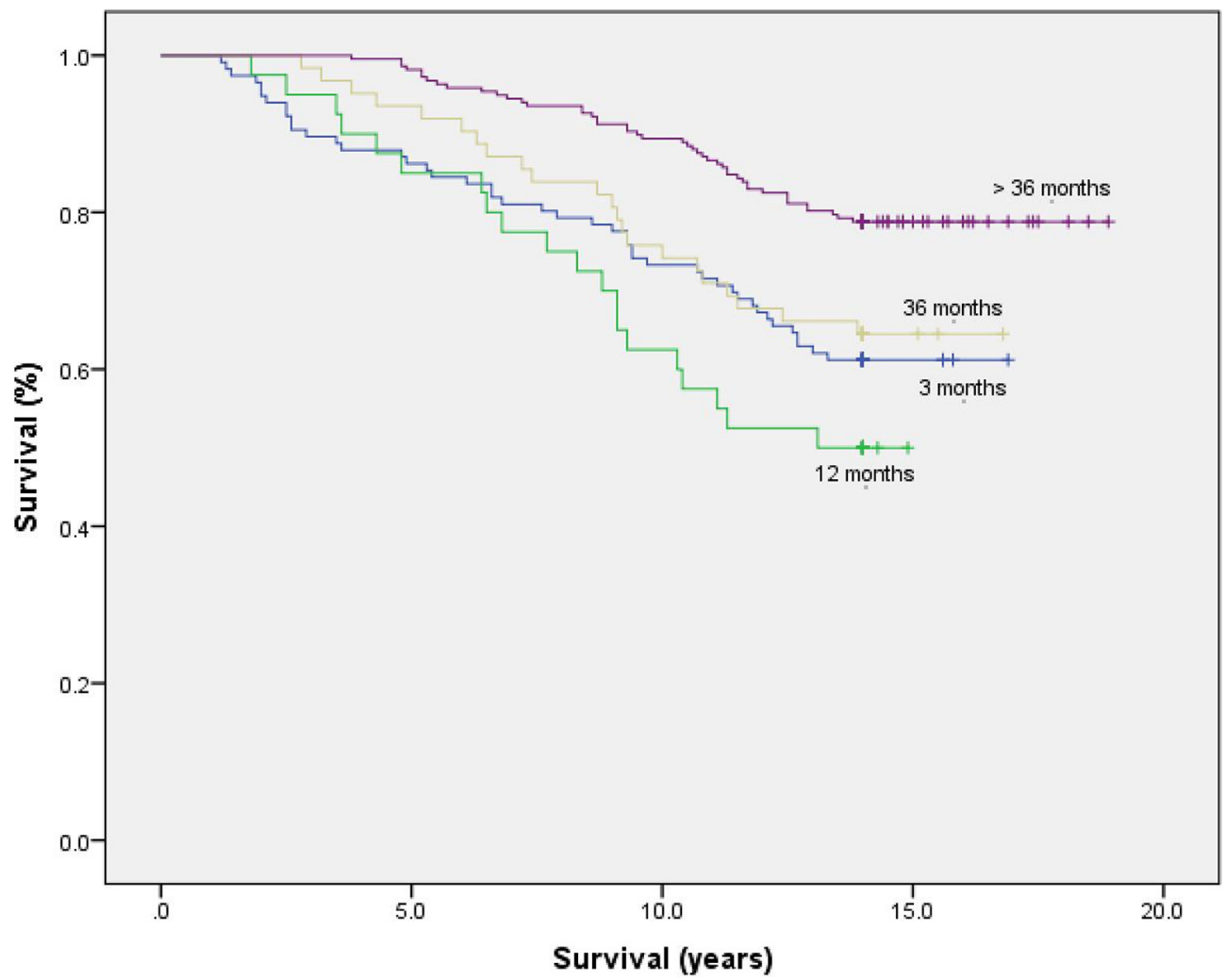

Figure 2 Unadjusted Kaplan-Meier plot comparing CR participants stratified by duration in the programme $(3,12$ and 36 month cutpoints).

29\% (multivariate-adjusted HR: >3 years follow-up: $0.71 ; 95 \%$ CI: 0.45 to $1.12 ; \mathrm{p}=0.142$ ). To further account for survivor selection bias among those attending $>36$ months of CR, Cox regression analysis was repeated with long-term programme duration as a time-dependent covariate. Following multivariate adjustment for the strongest predictors of all-cause mortality, long-term adherence to supervised CR exercise remained predictive of mortality risk reduction (table 2 ).

\section{DISCUSSION}

This study evaluated the impact of exercise 'dose' (volume expressed as MET-h per week) in a CR cohort undergoing long-term, exercise-based rehabilitation. Consistent with previous observational studies ${ }^{34}$ and recent meta-analysis, ${ }^{7}$ we found that a higher dose of exercise training was not associated with a significantly lower risk of all-cause mortality. Other prognostic factors, namely, cardiorespiratory fitness level at entry and extended CR programme adherence were more important predictors of mortality over 14 years. Though, importantly, exercise dose groups did not differ significantly with respect to overall programme adherence. A key finding was that longerterm ( $>36$ months) supervised CR exercise was associated with a significant stepwise survival benefit compared with all lesser durations. The findings herein therefore support the case for supervised, open-ended exercise training programmes within the community setting for long-term survival benefit.

In contrast to other large retrospective analyses of healthcare databases ${ }^{12}$ we did not find an independent protective effect of exercise dose in the long-term. In older propensity-matched patients, Suaya and colleagues ${ }^{2}$ found a significant difference in the cumulative mortality

Table 2 Estimated hazard ratios $(95 \% \mathrm{Cl})$ for all-cause mortality based on long-term ( $>36$ months) supervised CR exercise in time-dependent Cox regression analysis

\begin{tabular}{llll}
\hline & \multicolumn{2}{l}{ Long-term (>36 months) adherence to CR } & P Value \\
\cline { 2 - 4 } Model & HR $^{*}$ & $\mathbf{9 5 \%} \mathbf{~ C l}$ & 0.015 \\
\hline Unadjusted & 0.64 & 0.45 to 0.92 & 0.006 \\
Age-adjusted & 0.60 & 0.42 to 0.87 & 0.033 \\
Multivariable Model & 0.67 & 0.47 to 0.97 &
\end{tabular}

${ }^{*} \mathrm{HR}$ for $>36$ months $\mathrm{CR}$ ( $\leq 36$ months is reference). Multivariable model adjusted for: age, baseline sCRF, modified Charlson comorbidity index and secondary prevention medications (diuretic and ACE-inhibitor). 
rates of low ( 1 to 24 sessions) and high (25+ sessions) CR users after 5 years. ${ }^{2}$ Similarly, among older Medicare beneficiaries, Hammill and co-investigators ${ }^{1}$ observed that patients who participated in 36 sessions (maximum available) had a significantly lower risk of death at 4 years than those who attended fewer sessions. ${ }^{1}$ Potential explanations for such disparities between cohorts are likely to be due to length patient follow-up, minimum CR attendance requirements, age and overall burden of comorbidities in earlier cohorts.

Not all studies have demonstrated a beneficial effect of CR exercise dose on mortality ${ }^{4}$ and our results are consistent with other observations reporting longerterm follow-up. ${ }^{35}$ In $~ 5000$ propensity-matched patients referred to the Cardiac Wellness Institute, Calgary, Canada for 12 weeks CR, Martin and co-investigators (2013) demonstrated that each additional CR session attended was associated with a $1 \%$ decrease in mortality (unadjusted HR $0.9995 \%$ CI 0.98 to 0.99 ) over 5 years ${ }^{4}$. This association was lost when restricting analysis to patients completing a minimum of 12 weeks CR. Over comparable long-term follow-up to the present study, Beauchamp and co-investigators (2013) reported that attending $<25 \%$ of available CR sessions of low-moderate intensity exercise over six to eight sessions was associated with a mortality risk twice that of patients attending $\geq 75 \%$ of sessions, though this association was lost following adjustment for smoking status.

Overall, it appears that existing data do not support a clear or consistent strength of dose-response relationship between supervised CR exercise and all-cause mortality risk. Indeed, the heterogeneous character of programmes and absence of reported exercise prescriptions impedes its interpretation. Although a recent comprehensive review of high-quality RCT evidence recently found no significant reduction in all-cause mortality (relative risk: 1.01 ; $95 \%$ CI 0.89 to 1.15 ) with a higher dose of exercise-based $\mathrm{CR},{ }^{7}$ the short-term follow-up (median 12 months) and the quantification of dose by only duration and frequency, is likely to be inherently limited for the evaluation of this relationship. To the best of our knowledge, our study is the first to examine the effect of an extended length, exercise-rehabilitation programme (>36 months) on long-term mortality outcomes. Early trial findings from the National Exercise and Heart Disease Project, reported reductions in all-cause mortality ranging from $8 \%$ to $14 \%$ per 1-MET increment in CRF following 6 months supervised exercise training. ${ }^{22}$

Our finding that short-term (up to 3 months) and longterm CR adherers ( $>36$ months) did not appear to differ with respect to all-cause mortality risk may be explained in part, by sociodemographic factors. Short-term adherers were younger and had the highest percentage of working age participants in the cohort overall $(53 \%)$. Working status has been shown to be an important participation barrier to $\mathrm{CR},{ }^{23}$ which may support the contention that these participants left CR earlier to follow their own exercise training or habitual physical activity programme. ${ }^{24}$
Another noteworthy finding from the present cohort was that half of all participants undertaking a lower dose of exercise during CR were among the lowest fit at programme entry and undertook, on average, one fewer CR exercise session per week compared with those undertaking a higher dose. This suggests a 'self-selection' of a lower exercise dose by participants at the lower end of the fitness distribution. We have recently reported ${ }^{10}$ a $27 \%$ relative risk reduction in all-cause mortality per 1-MET gain achieved during CR for those with low fitness levels. Therefore, if the long-term secondary preventative benefits of exercise rehabilitation are to be attained then continued exercise supervision of lower fit individuals within extended community-based programmes may be beneficial.

Strengths of the present study include the length of complete follow-up for all-cause mortality including CR non-completers, detailed information on covariates (including smoking history and secondary prevention medications), extended multivariate Cox regression modelling including time-varying covariates, and the quantification of frequency, duration and intensity for the assessment of exercise dose.

\section{Limitations}

Although we have attempted to address the most common biases it is possible that the association between CR programme duration and mortality may be confounded by unmeasured or other 'healthy-adherer' behaviours. ${ }^{25}$ Given the importance of CRF on the prognosis of patients with $\mathrm{CHD}^{17}$ it is a limitation that fitness measures were not available for all patients after they left the programme. Though we included covariate adjustment for propensity score in our Cox regression models, the propensity score matching methodology has been suggested to provide the most precise estimate of 'treatment' effect. However, matching does not perform well when the number of 'treated' subjects is larger than the number of 'untreated' subjects, ${ }^{26}$ as was the case in our cohort. The lower dose of CR exercise associated with cycle ergometer testing may indicate an influence of test modality ${ }^{27}$ on long-term outcomes however, exercise mode was not a significant univariate predictor of mortality in the cohort overall.

The inability to report actual training heart rates from supervised CR exercise to quantify exercise dose is a limitation common to large observational cohorts, but may explain the lack of observed association between exercise dose and all-cause mortality risk in this study. Further dose-response analyses in large well-controlled studies of supervised exercise training over medium and long-term follow-up are therefore required to quantify any dose-related improvements in clinical outcomes. Future studies should aim to capture an overall measure of exercise dose (including exercise intensity, frequency and duration components), or corresponding cardiorespiratory fitness level, which could serve as the basis of a minimal dose recommendation for clinical benefit. 
Finally, it is noted that our finding that certain cardioprotective medications, notably ACE-inhibitor and diuretic therapies were independent adverse predictors of all-cause mortality is contrary to strong RCT evidence. ${ }^{28}$ Findings may reflect differences in underlying disease severity, prevalence of co-morbidities and self-selection of low volume exercise training.

\section{CONCLUSION}

The results of this study suggest that long-term supervised exercise rehabilitation ( $>36$ months) is associated with a markedly lower risk of all-cause mortality and supports the benefits of continued exercise supervision within community-based CR programmes.

Contributors CTa (corresponding author), CT, KW, SC, LI contributed to the study design. CTa, SC, CT, JM, JWM and MD collected the data. CTa, LI, SC involved in editing and data preparation. CTa performed the data analysis. CTa, SC and LI contributed to the first draft of the manuscript. CT, SC, LI, read and critically appraised several versions of the manuscript. All authors have critically revised the manuscript for important intellectual content and provided final approval of the version for publication.

Funding This research received no specific grant from any funding agency in the public, commercial or not-for-profit sectors.

Competing interests None declared.

Ethics approval Approval for this study was granted in 2013 from Yorkshire and the Humber (Leeds East) Local Research Ethics Committee (reference 13/YH/0099) and the Health Research Authority Confidentiality Advisory Group (reference CAG 5-03(PR6)/2013).

Provenance and peer review Not commissioned; externally peer reviewed.

Open Access This is an Open Access article distributed in accordance with the Creative Commons Attribution Non Commercial (CC BY-NC 4.0) license, which permits others to distribute, remix, adapt, build upon this work non-commercially, and license their derivative works on different terms, provided the original work is properly cited and the use is non-commercial. See: http://creativecommons.org/ licenses/by-nc/4.0/

C Article author(s) (or their employer(s) unless otherwise stated in the text of the article) 2017. All rights reserved. No commercial use is permitted unless otherwise expressly granted.

\section{REFERENCES}

1. Hammill BG, Curtis LH, Schulman KA, et al. Relationship between cardiac rehabilitation and long-term risks of death and myocardial infarction among elderly Medicare beneficiaries. Circulation 2010;121:63-70.

2. Suaya JA, Stason WB, Ades PA, et al. Cardiac rehabilitation and survival in older coronary patients. J Am Coll Cardiol 2009;54:25-33.

3. Pack QR, Goel K, Lahr BD, et al. Participation in cardiac rehabilitation and survival after coronary artery bypass graft surgery: a community-based study. Circulation 2013;128:590-7.

4. Martin BJ, Hauer T, Arena R, et al. Cardiac rehabilitation attendance and outcomes in coronary artery disease patients. Circulation 2012;126:677-87.

5. Beauchamp A, Worcester M, Ng A, et al. Attendance at cardiac rehabilitation is associated with lower all-cause mortality after 14 years of follow-up. Heart 2013;99:620-5.
6. Heran BS, Chen JM, Ebrahim S, et al. Exercise-based cardiac rehabilitation for coronary heart disease. Cochrane Database Syst Rev 2011:CD001800.

7. Anderson L, Oldridge N, Thompson DR, et al. Exercise-Based Cardiac Rehabilitation for Coronary Heart disease: cochrane Systematic Review and Meta-Analysis. J Am Coll Cardiol 2016:67:1-12.

8. Uddin J, Zwisler AD, Lewinter C, et al. Predictors of exercise capacity following exercise-based rehabilitation in patients with coronary heart disease and heart failure: A meta-regression analysis. Eur J Prev Cardiol 2016;23:683-93.

9. Barons MJ, Turner S, Parsons N, et al. Fitness predicts long-term survival after a cardiovascular event: a prospective cohort study. BMJ Open 2015;5:e007772.

10. Taylor C, Tsakirides C, Moxon J, et al. Submaximal fitness and mortality risk reduction in coronary heart disease: a retrospective cohort study of community-based exercise rehabilitation. BMJ Open 2016:6:e011125.

11. Martin BJ, Arena R, Haykowsky M, et al.Cardiovascular fitness and mortality after contemporary cardiac rehabilitation. Mayo Clin Proc 2013;88:455-63.

12. Almodhy M, Ingle L, Sandercock GR. Effects of exercise-based cardiac rehabilitation on cardiorespiratory fitness: a meta-analysis of UK studies. Int J Cardiol 2016;221:644-51.

13. Lehmann G, Schmid S, Ammer R, et al. Evaluation of a new treadmil exercise protocol. Chest 1997;112:98-106.

14. Pescatello LS, Arena R, Riebe D, et al. Guidelines for exercise testing and prescription. 9th ed. Baltimore: Lippincott Williams \& Wilkins, 2010.

15. Givoni B, Goldman RF. Predicting metabolic energy cost. J App/ Physiol 1971;30:429-33.

16. Astrand PO, Ryhming I. A nomogram for calculation of aerobic capacity (physical fitness) from pulse rate during sub-maximal work. $J$ Appl Physiol 1954;7:218-21.

17. Myers J, Prakash M, Froelicher V, et al. Exercise capacity and mortality among men referred for exercise testing. $N$ Engl $J$ Med 2002;346:793-801.

18. D'Hoore W, Bouckaert A, Tilquin C. Practical considerations on the use of the Charlson comorbidity index with administrative data bases. J Clin Epidemiol 1996;49:1429-33.

19. Wannamethee SG, Shaper AG, Walker M. Physical activity and mortality in older men with diagnosed coronary heart disease. Circulation 2000;102:1358-63.

20. Therneau TM, Grambsch PM. Modeling survival data: extending the cox model. New York: Springer-Verlag, 2000.

21. Suissa S. Immortal time bias in pharmaco-epidemiology. Am J Epidemiol 2008;167:492-9.

22. Dorn J, Naughton J, Imamura D, et al. Results of a multicenter randomized clinical trial of exercise and long-term survival in myocardial infarction patients: the National Exercise and Heart Disease Project (NEHDP). Circulation 1999;100:1764-9.

23. Clark AM, King-Shier KM, Spaling MA, et al. Factors influencing participation in cardiac rehabilitation programmes after referral and initial attendance: qualitative systematic review and meta-synthesis. Clin Rehabil 2013;27:948-59.

24. Rivett MJ, Tsakirides C, Pringle A, et al. Physical activity readiness in patient withdrawals from cardiac rehabilitation. $\mathrm{Br} J$ Nurs 2009;18:188-91.

25. Alter DA, Zagorski B, Marzolini S, et al. On-site programmatic attendance to cardiac rehabilitation and the healthy-adherer effect. Eur J Prev Cardiol 2015;22:1232-46.

26. Austin PC. The use of propensity score methods with survival or time-to-event outcomes: reporting measures of effect similar to those used in randomized experiments. Stat Med 2014;33:1242-58.

27. Myers J, Buchanan N, Walsh D, et al. Comparison of the ramp versus standard exercise protocols. J Am Coll Cardio 1991;17:1334-42.

28. Wright JM, Musini VM. First-line drugs for hypertension. Cochrane Database Syst Rev 2009. 